\title{
Avaliação do Uso de Amidos Aniônicos Hidrolisados como Estabilizantes em Nanocápsulas Poliméricas para Formulações Tópicas
}

\author{
Ester P. dos Santos, Jayne C. S. Barboza \\ Departamento de Engenharia Química, USP
}

\begin{abstract}
Resumo: A proposta deste trabalho foi introduzir amidos modificados (aniônicos) hidrolisados como tensoativos em uma formulação para a encapsulação da benzofenona-3 com uma matriz polimérica de poli( $\varepsilon$-caprolactona) (PCL), avaliando a influência deste componente nas características físico-químicas das nanopartículas. As nanopartículas foram preparadas com a técnica de deposição interfacial do polímero pré-formado, empregando-se um planejamento de experimentos de mistura. Previamente, os amidos foram hidrolisados via processo enzimático e medidas de tensão superficial foram realizadas para avaliar a capacidade tensoativa das soluções aquosas dos amidos hidrolisados para aplicação nas formulações de nanocápsulas. Os resultados indicaram que os amidos avaliados podem ser empregados como tensoativos nas formulações, sem alterar significativamente propriedades, como diâmetro, polidispersão e percentual de encapsulação.
\end{abstract}

Palavras-chave: Amidos aniônicos, tensoativo, nanocápsulas, benzofenona-3.

\section{Evaluation of Anionic Hydrolyzed Starch as Stabilizers in Polymeric Nanocapsules for Topical Formulations}

\begin{abstract}
The purpose of this work was to introduce modified hydrolyzed starches (anionic) as surfactant in a formulation for the encapsulation of benzophenone- 3 with a polymer matrix of poly( $\varepsilon$-caprolactone) (PCL), including the study of the influence from the surfactant on the physicochemical characteristics of nanoparticles. The nanoparticles were prepared via interfacial deposition of the pre-formed polymer, using an experiment design for the mixtures. The starches were initially hydrolyzed via an enzymatic process and surface tension measurements were performed to assess the ability of surfactant aqueous solutions of hydrolyzed starch for application in nanocapsule formulations. The results indicated that the starches can be employed as surfactant in the formulations, without significantly altering properties such as diameter, polydispersity and encapsulation efficiency.
\end{abstract}

Keywords: Anionic starches, surfactant, nanocapsules, benzophenone-3.

\section{Introdução}

A presença de macromoléculas em dispersões coloidais pode fornecer tanto um efeito de estabilização quanto de desestabilização. A estabilização ocorre devido ao aumento da viscosidade da fase contínua causada pela presença destas macromoléculas, mas também pode ser atribuída à adsorção das macromoléculas na superfície das partículas coloidais. Por outro lado, a desestabilização pode ocorrer se a camada superficial adsorvida é insuficiente. As macromoléculas não adsorvidas ficarão, então, nas proximidades da superfície da fase dispersa, causando a floculaçãa ${ }^{[1]}$. Compostos de alta massa molecular, por exemplo, polissacarídeos podem proporcionar uma estabilização estérica em sistemas dispersos.

Dentre os polissacarídeos existentes, o amido tem despertado um grande interesse como agente estabilizante. Um número considerável de trabalhos aponta a atuação de amidos modificados como agentes estabilizantes em sistemas dispersos, principalmente em emulsões ${ }^{[1-5]}$. Porém, as aplicações dos amidos nativos são limitadas devido a sua instabilidade durante o armazenamento. Por esta razão, foram desenvolvidas técnicas para modificá-los, aumentando assim suas propriedades e aplicações.

A atividade tensoativa dos amidos é obtida, por exemplo, através de modificação química a qual introduz grupos hidrofóbicos em sua estrutura polimérica. Uma das técnicas de modificação química é o tratamento destes amidos com anidrido octenil succínico, obtendo assim os chamados amidos $\mathrm{OSA}^{[6]}$. Esta modificação fornece um caráter anfifílico à molécula de amido e, consequentemente, propriedades ativas de superfície. Devido ao seu alto peso molecular e estrutura ramificada, o amido modificado adsorvido à interface é capaz de estabilizar emulsões e outros sistemas dispersos por impedimento estérico a baixas concentrações ${ }^{[3]}$. 
Devido ao comportamento anfifílico das moléculas dos amidos modificados, algumas técnicas são necessárias para a determinação de suas características ativas de superfície como, por exemplo, a concentração micelar crítica (CMC). Visando determinar a CMC de dois tipos de amidos OSA e suas interações com goma xantana, Krstonosić et al. ${ }^{[7]}$, utilizaram viscosimetria, condutimetria, tensão superficial e colorimetria para verificar a capacidade tensoativa destes componentes. De acordo com os resultados apresentados, os amidos OSA reduziram significativamente a tensão superficial da água, assim como as respectivas misturas com goma xantana confirmando sua aplicabilidade como agente ativo de superfície em um sistema emulsionado.

Nanopartículas poliméricas biodegradáveis possuem um grande interesse na comunidade científica devido às suas potencialidades terapêuticas, à maior estabilidade nos fluídos biológicos e durante o armazenamento. São definidas como sistemas carreadores de fármacos e moléculas ativas que apresentam diâmetro de 10 a $100 \mathrm{~nm}$. De acordo com sua composição/organização estrutural e método de preparação podem ser classificadas em nanocápsulas (NC) e nanoesferas (NE). As NC são compostas por uma matriz polimérica disposta ao redor de um núcleo oleoso, podendo o ativo (droga) estar dissolvido neste núcleo oleoso e/ou adsorvido à parede polimérica. Já as NE, por sua vez, não apresentam óleo em sua composição e são constituídas por um invólucro polimérico, no qual o ativo pode ficar retido ou adsorvido $^{[8,9]}$

Uma aplicação de grande interesse das nanopartículas poliméricas é na área cosmética como veículos tópicos, principalmente para fotoprotetores, com a intenção de prolongar o tempo de resistência dos filtros no estrato córneo, assim como evitar o processo de fotodegradação mantendo sua estabilidade ${ }^{[10-12]}$. Por apresentarem um tamanho reduzido, estes sistemas carreadores possibilitam um maior contato das moléculas ativas com o estrato córneo, além de facilitar sua formulação em produtos dermatológicos e propiciar confortável aplicação cutânea $^{[13,14]}$. De acordo com Sinha et al. ${ }^{[15]}$, a liberação do ativo, a degradação e estabilidade físico-química das NP podem ser moduladas com a escolha adequada de seus componentes, potencializando suas aplicações.

Dentre os componentes de uma formulação de nanopartículas poliméricas, os tensoativos são fundamentais para impedir sua agregação, após o preparo e durante o armazenamento por promoverem a estabilização física e/ou química da suspensão. Os tensoativos mais utilizados são os de alta hidrofilia não iônicos como os polisorbatos (Tween ${ }^{\circledR}$ ) e, de acordo com o método empregado para obtenção de nanopartículas, também são utilizados tensoativos de baixa hidrofilia não iônicos como os ésteres de sorbitana $\left(\operatorname{Span}^{\circledR}\right)^{[16,17]}$.

Considerando a potencialidade dos amidos modificados atuarem como agentes estabilizantes, principalmente em emulsões, o objetivo deste trabalho foi introduzir amidos modificados aniônicos, previamente hidrolisados, como agentes tensoativos no sistema de nanopartículas poliméricas e avaliar sua influência nas características físico-químicas das mesmas.

\section{Experimental}

\section{Materiais e métodos}

\section{Solventes e reagentes}

O amido modificado aniônico utilizado neste trabalho foi o Superion 3050, com grau de substituição DS $=0,045 \mathrm{~mol} / \mathrm{mol}$, cedido pela empresa Novo Horizonte. Os amidos aniônicos hidrolisados aplicados nas formulações foram denominados como: Amido Aniônico de Alta Massa Molar (AAAMM), com massa molar ponderal média $\left(\overline{M_{w}}\right)$ de $2952,2 \mathrm{~g} / \mathrm{mol}$, massa molar numérica média $\left(\overline{M_{n}}\right)$ de $1330,0 \mathrm{~g} / \mathrm{mol}$ e índice de polidispersão (IP) igual a 2,22; e Amido Aniônico de Baixa Massa Molar (AABMM) com $\overline{M_{w}}=1650,5 \mathrm{~g} / \mathrm{mol}$, $\overline{M_{n}}=850,7 \mathrm{~g} / \mathrm{mol}$ e IP $=1,94$. A matriz polimérica, poli( $\varepsilon$-caprolactona) (PCL) foi sintetizada em laboratório, via polimerização em massa ${ }^{[18]}$ e apresentou os seguintes valores massas molares e polidispersão: $\overline{M_{n}}=45654 \mathrm{~g} / \mathrm{mol}, \overline{M_{w}}=69926 \mathrm{~g} / \mathrm{mol} \mathrm{e} \mathrm{IP}=1,53$. Para a preparação das nanopartículas empregaram-se Miglyol $810^{\circledR}$ (mistura de triglicerídeos dos ácidos cáprico e caprílico - Delaware); Benzofenona-3 (2-hidróxi-4-metóxi-benzofenona - Aldrich) e Span $60^{\circledR}$ (monoestearato de sorbitano - Aldrich). O tensoativo sintético da formulação, Alkest TW $80 \mathrm{~K}^{\circledR}$ (polisorbato 80), foi fornecido pela empresa Oxiteno. Os demais produtos químicos e todos os solventes foram utilizados sem tratamento prévio para purificação por apresentarem grau de pureza analítico.

\section{Medidas de tensão superficial dos amidos hidrolisados}

As medidas de tensão superficial foram conduzidas em um tensiômetro de anel 2-Hamburg 39 (Kruss) (ASTM D 1331-56). Para realização das análises, foram preparadas soluções aquosas dos amidos hidrolisados nas seguintes concentrações: 0,$1 ; 0,25 ; 0,50$ e $1,0 \%$ $\mathrm{m} / \mathrm{m}$. Todas as medidas foram realizadas em triplicata, mantendo-se a temperatura constante em $25^{\circ} \mathrm{C} \pm 0,1^{\circ} \mathrm{C}$.

\section{Preparação das nanocápsulas poliméricas}

As suspensões de nanopartículas poliméricas contendo BZ-3 foram preparadas de acordo com o método de deposição interfacial de polímeros pré-formados ${ }^{[19]}$. $\mathrm{O}$ filtro químico foi dissolvido na fase orgânica composta por Miglyol $810^{\circledR}$, Span $60^{\circledR}$, PCL e acetona. A fase orgânica foi vertida sobre a fase aquosa contendo Alkest TW $80 \mathrm{~K}^{\circledR}$, amidos hidrolisados e água Milli-Q ${ }^{\circledR}$, através de um funil de adição e mantida sob agitação magnética durante $10 \mathrm{~min}$. As formulações foram preparadas ao abrigo da luz e evaporadas em rotavapor sob a temperatura de $35{ }^{\circ} \mathrm{C}$ até completa eliminação do solvente. Todas as formulações foram diluídas em balão volumétrico de 100,0 mL com água Milli-Q ${ }^{\circledR}$ e preparadas em duplicata. A Tabela 1 apresenta as proporções dos reagentes utilizadas nas formulações.

Para avaliar os efeitos quantitativos da adição de amidos hidrolisados como agentes tensoativos da fase aquosa foi utilizado um planejamento experimental de misturas do tipo simplex. Neste planejamento a fração molar do tensoativo hidrofílico (Alkest TW $80 \mathrm{~K}^{\circledR}$ ) foi 
Tabela 1. Formulação referência para o preparo das nanocápsulas poliméricas $^{[20]}$.

\begin{tabular}{lll}
\hline \multicolumn{1}{c}{ Fase } & \multicolumn{1}{c}{ Componente } & \multicolumn{1}{c}{ Quantidade $(\mathbf{m g})$} \\
\hline Orgânica & PCL & 100 \\
& Miglyol $810^{\circledR}$ & 313,5 \\
& Span $60^{\circledR}$ & 76,6 \\
& BZ-3 & 50 \\
& Acetona & 31600 \\
Aquosa & Água Milli-Q ${ }^{\circledR}$ & 53300 \\
& Polisorbato 80 & 76,6 \\
\hline
\end{tabular}

variada, a qual foi substituída pelos amidos hidrolisados, mantendo-se constante o número de mol final de tensoativos da fase aquosa ${ }^{[21]}$. As análises estatísticas foram realizadas utilizando-se o software Minitab 15.

\section{Caracterização físico-química das formulações}

A caracterização físico-química das formulações foi realizada imediatamente após a preparação. As formulações foram avaliadas com relação aos aspectos macroscópicos, tamanho de partículas, polidispersão, $\mathrm{pH}$ e percentual de encapsulação de benzofenona-3.

\section{Análise macroscópica}

Cada formulação $(3,0 \mathrm{~mL})$ foi mantida em tubo de vidro transparente e avaliada visualmente com relação à cor, odor, presença de precipitados ou separação de fases, sob luz natural.

\section{Análises de pH}

As medidas de $\mathrm{pH}$ foram efetuadas diretamente nas amostras utilizando-se um pHmetro FE20/EL20 (Mettler Toledo), previamente calibrado com soluções tampão para calibração pH 4,0 e 7,0 (Merck).

\section{Determinação do tamanho de partículas e polidispersão}

O diâmetro médio e a polidispersão das nanopartículas em suspensão foram determinados através de espalhamento de luz utilizando-se um equipamento Zetasizer 1000 (Malvern Instruments). As amostras foram diluídas na proporção 1:50 (v/v) em água Milli-Q ${ }^{\circledR}$ e a distribuição de tamanho foi avaliada efetuando-se três varreduras com 10 leituras cada.

Extração líquido-líquido da benzofenona-3, BZ-3, não encapsulada

A BZ-3 não encapsulada (livre) foi removida da suspensão a partir da técnica de extração líquido-líquido, empregando-se hexano como solvente (não solvente do polímero). A fração extraída (hexano/benzofenona-3) foi tratada com sulfato de sódio anidro para remoção de vestígios de água. Esta solução foi filtrada e recolhida em um frasco limpo e seco para posterior caracterização.

Determinação do percentual de encapsulação da benzofenona-3 nas nanocápsulas

Para a quantificação da BZ-3 livre, as soluções de BZ-3 em hexano foram secas no rotavapor para total eliminação do solvente. Em seguida, toda a massa contida no frasco foi dissolvida com acetonitrila e, posteriormente, transferida para um balão volumétrico de 50,0 mL. A quantidade do ativo não encapsulado foi determinada em um espectrômetro de UV/VIS (Perkin Elmer) em $289 \mathrm{~nm}$. O percentual de encapsulação (E) foi determinado a partir da razão entre a diferença da concentração total de benzofenona-3 na suspensão (CT) e a concentração de benzofenonas-3 livre (CL) e a concentração total, multiplicada por $100^{[19]}$ (Equação 1):

$$
E=\frac{C T-C L}{C T} \times 100
$$

\section{Resultados e Discussão}

\section{Tensão superficial dos amidos aniônicos hidrolisados}

Um dos parâmetros mais importantes para designar a aplicação de um determinado composto como agente tensoativo é sua tensão superficial. Pode-se dizer que a maioria das propriedades dos agentes tensoativos depende de sua capacidade em reduzir a tensão superficial, pois é a relação entre a estrutura química, a velocidade e o grau de adsorção na interface ou superfície, que diferencia os vários tipos de tensoativos e, consequentemente, determina sua aplicação.

A capacidade tensoativa de cada amido foi avaliada plotando-se gráficos de tensão superficial versus concentração. Estes resultados para os dois amidos aniônicos AAAMM e AABMM estão apresentados nas Figuras 1a, b, respectivamente.

De acordo com os testes tensão superficial, os amidos AAAMM (Figura 1a) e AABMM (Figura 1b) reduziram a tensão superficial daágua. Krstonosić et al. ${ }^{[7]}$ determinaram a CMC de dois diferentes amidos modificados (OSA) por viscosimetria, condutimetria, tensão superficial e colorimetria. Os valores da CMC para os dois amidos OSA (OS1 e OS2) variaram de 0,050 a $0,088 \mathrm{~g} / 100 \mathrm{~cm}^{3}$ e de 0,041 a $0,081 \mathrm{~g} / 100 \mathrm{~cm}^{3}$, respectivamente, dependendo da técnica aplicada. Nos testes de tensão foi observada a capacidade dos amidos OSA em diminuir significativamente a tensão superficial da água, isto é, a capacidade tensoativa da molécula. Segundo os autores, os amidos OSA estudados podem atuar como agentes tensoativos em um sistema de emulsão O/A. Analogamente, de acordo com os resultados obtidos, ambos os amidos podem atuar como agentes ativos de superfície no sistema de nanopartículas poliméricas por apresentarem uma redução significativa na tensão superficial. Segundo os resultados, o amido hidrolisado AAAMM reduziu a tensão superficial da água de $74 \mathrm{~m} . \mathrm{N} / \mathrm{m}$ para $61,8 \mathrm{~m} . \mathrm{N} / \mathrm{m}$ (por volta de $17 \%$ ) e o amido hidrolisado AABMM de $74 \mathrm{~m} . \mathrm{N} / \mathrm{m}$ para $56,5 \mathrm{~m} . \mathrm{N} / \mathrm{m}$ (aproximadamente 24\%), ambos para as soluções aquosas contendo $1,0 \% \mathrm{~m} / \mathrm{m}$ dos respectivos amidos.

\section{Caracterização físico-química das formulações de nanopartículas poliméricas}

\section{Análise macroscópica}

Após o preparo, as suspensões de nanocápsulas apresentaram aparência macroscópica homogênea, 


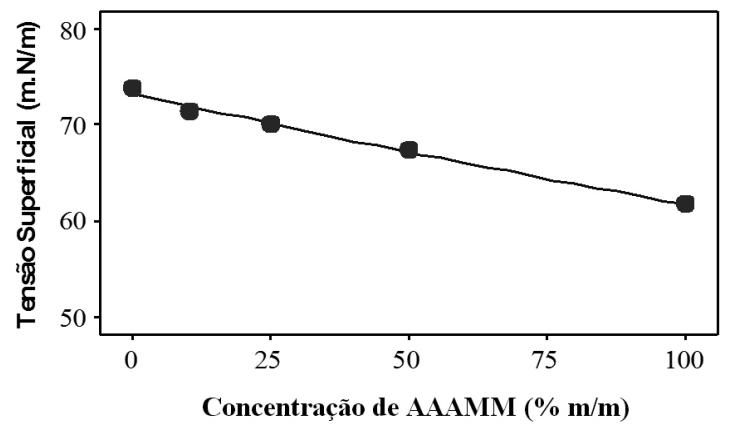

(a)

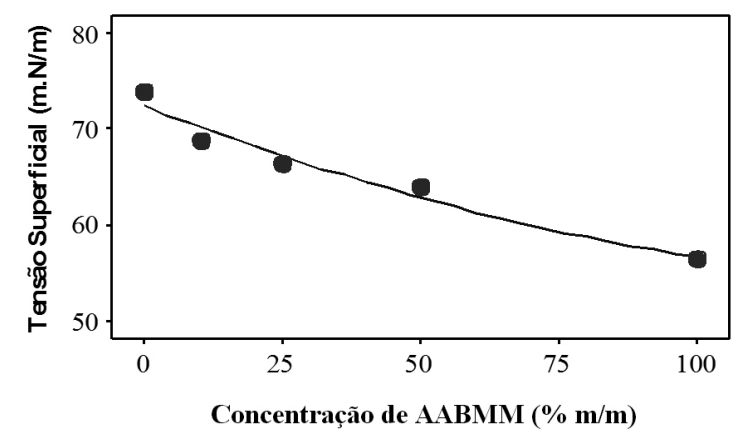

(b)

Figura 1. Tensão superficial versus concentração para os amidos aniônicos hidrolisados: (a) Amido Aniônico de Alta Massa Molar e (b) Amido Aniônico de Baixa Massa Molar.

aspecto leitoso, branco e opalescente. As suspensões exibiram reflexo azulado, devido ao movimento browniano das nanopartículas em suspensão. O aspecto visual das suspensões de nanocápsulas foi semelhante ao descrito na literatura para diferentes sistemas de nanocápsulas obtidas pelo método de deposição interfacial de polímeros pré-formados ${ }^{[11,20,22-24]}$.

\section{Análises de pH}

Todas as formulações de poli( $\varepsilon$-caprolactona) apresentaram $\mathrm{pH}$ ácido $(\mathrm{pH}=5,0)$, devido à presença do grupo éster, o qual sofrerá hidrólise ao longo do tempo, reduzindo ainda mais o valor de $\mathrm{pH}^{[25]}$. Suspensões de nanocápsulas de PCL contendo outros ativos também exibiram caráter ácido ${ }^{[17,20,26]}$. De acordo com Alves et al. [13], as suspensões produzidas encontram-se coerentes para este tipo de sistema e na faixa de $\mathrm{pH}$ adequada para formulações de uso tópico (3 a 10), não interferindo nas características de permeação de moléculas lipofílicas, como é o caso da BZ-3.

\section{Avaliação dos parâmetros diâmetro de partículas, polidispersão e percentual de encapsulação}

Todas as formulações apresentaram partículas com diâmetros entre 100 e $300 \mathrm{~nm}$. Os diâmetros médios obtidos estão de acordo com os dados relatados na literatura para nanocápsulas formadas pelo método de deposição interfacial de polímeros pré-formados ${ }^{[11,20,24]}$. A Figura 2 mostra o efeito dos amidos modificados aniônicos hidrolisados sobre o diâmetro médio de partículas.

Um parâmetro muito importante que afeta as propriedades das nanopartículas é a característica (natureza e concentração) do agente tensoativo empregado na formulação. O aumento da concentração deste componente pode tanto aumentar, quanto diminuir o diâmetro das nanopartículas, dependendo de sua natureza ${ }^{[16]}$.

A Tabela 2 apresenta os efeitos de cada fator (amidos aniônicos hidrolisados e polisorbato 80) em relação ao diâmetro médio de partículas, polidispersão e percentual de encapsulação.

A análise da Tabela 2 mostra que a menor contribuição para o aumento do diâmetro médio de partículas foi devido aos componentes AABMM $(103 \pm 75 \mathrm{~nm})$ e

\section{POLISORBATO 80}
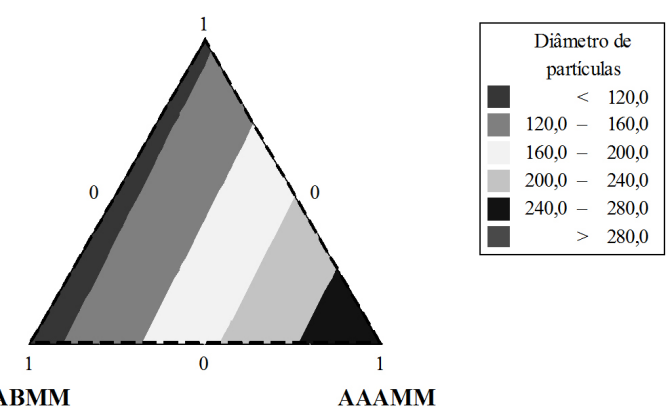

AABMM

AAAMM

Figura 2. Efeito dos amidos aniônicos sobre o diâmetro médio de partículas.

POLISORBATO $80(113 \pm 75 \mathrm{~nm})$, pois estes possuem os menores coeficientes.

Neste trabalho foi possível correlacionar a capacidade tensoativa dos amidos aniônicos hidrolisados, ou seja, a redução da tensão superficial da água com a variação nos diâmetros médios das nanocápsulas de BZ-3. Isto é, os amidos aniônicos os quais reduziram a tensão superficial contribuíram, de maneira não significativa, para o aumento do diâmetro de partículas. Assim como AAAMM que, apesar de reduzir pouco a tensão superficial, não contribuiu de maneira significativa para o aumento de DP.

De acordo com os resultados apresentados, os diâmetros das nanocápsulas de PCL/BZ-3 contendo amidos aniônicos hidrolisados foram adequados para aplicação de produtos tópicos, visto que os diâmetros recomendados para este objetivo são inferiores a $600 \mathrm{~nm}^{[11]}$.

Já em relação à polidispersão, a Tabela 2 mostra que os dois tipos de amidos aniônicos hidrolisados, AAAMM e AABMM, contribuem da mesma forma para o aumento da polidispersão.

$\mathrm{O}$ índice de polidispersão, calculado pelo equipamento, reflete o perfil de homogeneidade no tamanho das nanopartículas. Segundo a literatura, um índice de polidispersão até 0,3 é considerado adequado para aplicação cutânea ${ }^{[20]}$. Entretanto, índices de polidispersão altos refletem a ampla variação no tamanho das nanopartículas, isto é, uma falta de controle 
Tabela 2. Efeitos dos amidos aniônicos sobre diâmetro médio de partículas, polidispersão e percentual de encapsulação.

\begin{tabular}{lcccccc}
\hline \multirow{2}{*}{ Fator } & \multicolumn{2}{c}{ Efeito sobre o diâmetro de partícula } & \multicolumn{2}{c}{ Efeito sobre a polidispersão } & \multicolumn{3}{c}{ Efeito sobre o percentual de encapsulação } \\
\cline { 2 - 7 } & Valor $(\mathbf{n m})$ & Erro $(\mathbf{\pm n m})$ & Valor & Erro $( \pm)$ & Valor $(\boldsymbol{\%})$ & Erro $( \pm \%)$ \\
\hline POLISORBATO & 113 & 75 & 0,972 & 0,097 & 101 & 3 \\
AABMM & 103 & 75 & 0,831 & 0,097 & 100 & 3 \\
AAAMM & 281 & 75 & 0,856 & 0,097 & 101 & 3 \\
\hline
\end{tabular}

\section{POLISORBATO 80}

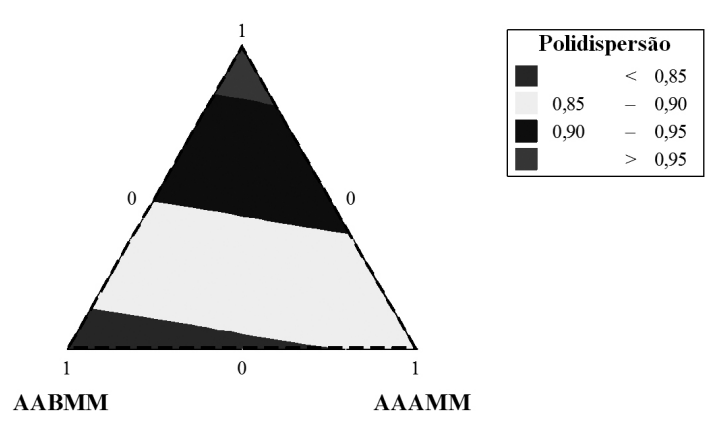

Figura 3. Efeito dos amidos aniônicos sobre a polidispersão.

nos diâmetros das partículas formadas, a qual pode ser afetada por diversos fatores: natureza e concentração do polímero e do fármaco, concentração de surfatantes, proporção entre solvente orgânico e água, concentração e natureza do óleo, além da velocidade de difusão da fase orgânica na aquosa. A Figura 3 mostra os efeitos dos amidos hidrolisados sobre a polidispersão.

O método também foi validado, afirmando que a variação da fração molar do tensoativo da fase aquosa não interfere na polidispersão, apresentando um coeficiente de correlação de $0,00 \%$. De acordo com o modelo, independente da fração molar de tensoativo da fase aquosa, a polidispersão apresentará sempre os mesmos valores.

Diversos fatores sãocapazes de influenciar a quantidade de ativo associada aos sistemas nanoestruturados, dentre os quais se destacam as características físico-químicas do ativo, o pH do meio, as características de superfície das partículas ou a natureza do polímero, a quantidade de ativo adicionada à formulação, a natureza do óleo utilizado (no caso das NCs) e o tipo de tensoativo adsorvido à superfície polimérica ${ }^{[8]}$.

Assim como nos resultados de polidispersão, todos os componentes contribuíram para o aumento do percentual de encapsulação, ou seja, os amidos AABMM $(100 \pm 3 \%)$ e AAAMM $(101 \pm 3 \%)$ e o tensoativo sintético POLISORBATO $80(101 \pm 3 \%)$. Como o erro experimental dos coeficientes foi de $\pm 3 \%$ não há diferença significativa entre eles, isto é, todas as formulações apresentaram um percentual de encapsulação maior que $95 \%$. O modelo indicou que a variação da fração molar do tensoativo da fase aquosa representa $97,8 \%$ do efeito sobre o percentual de encapsulação observado.

\section{Conclusão}

A introdução dos amidos aniônicos hidrolisados nas formulações não acarretou mudança significativa nas propriedades das nanocápsulas, principalmente com relação à estabilidade coloidal. Estes resultados apenas confirmam que os amidos aniônicos hidrolisados utilizados neste trabalho podem substituir o tensoativo sintético polisorbato 80, para a aplicação estudada, visando o desenvolvimento de novas formulações para protetores solares.

\section{Agradecimentos}

A CAPES pelo financiamento do projeto.

\section{Referências Bibliográficas}

1. Nilsson, L. \& Bergenståhl, B. - Langmuir, 22, p.8770 (2006). PMid:17014116. http://dx.doi.org/10.1021/ la060870f

2. Nilsson, L. \& Bergenståhl, B. - J. Ag. Food Chem., 55, p.1469 (2007). PMid:17243695. http://dx.doi.org/10.1021/ jf062087z

3. Tesch, S.; Gerhards, C. \& Schubert, H. - J. Food Eng., 54, p.167 (2002). http://dx.doi.org/10.1016/S02608774(01)00206-0

4. Varona, S.; Martín, A. \& Cocero, M. J. - Chem. Eng. Process., 48, p.1121 (2009). http://dx.doi.org/10.1016/j. cep.2009.03.002

5. Yusoff, A. \& Murray, B. S. - Food Hydrocoll., 25, p.42 (2011). http://dx.doi.org/10.1016/j.foodhyd.2010.05.004

6. Bhosale, R. \& Singhal, R. - Carbohyd. Polym., 66, p.521 (2006). http://dx.doi.org/10.1016/j.carbpol.2006.04.007

7. Krstonosić, V.; Dokić, L. \& Milanović, J. - Food Hydrocoll., 25, p.361 (2011).

8. Schaffazick, S. R.; Guterres, S. S.; Freitas, L. L. \& Pohlmann, A. R. - Quim. Nova, 26, p.726 (2003). http:// dx.doi.org/10.1590/S0100-40422003000500017

9. Soppimath, K. S.; Aminabhavi, T. M.; Kulkarni, A. R. \& Rudzinski, W. E. - J. Control. Release, 70, p.1 (2001). http://dx.doi.org/10.1016/S0168-3659(00)00339-4

10. Weiss-Angeli, V. - "Desenvolvimento e caracterização de formulações fotoprotetoras contendo nanocápsulas", Tese de Doutorado, Universidade Federal do Rio Grande do Sul, Brasil (2007)

11. Paese, K. - "Desenvolvimento tecnológico, estudo da fotoestabilidade e avaliação da permeação cutânea in vitro da benzofenona-3 a partir de nanocápsulas poliméricas incorporadas em diferentes veículos semi-sólidos", Dissertação de Mestrado, Universidade Federal do Rio Grande do Sul, Brasil (2008).

12. Alvarez-Román, R.; Naik, A.; Kalia, Y. N.; Guy, R. H. \& Fessi, H. - J. Control. Release, 99, p.53 (2004). PMid:15342180. http://dx.doi.org/10.1016/j. jconrel.2004.06.015 
13. Alves, M. P.; Scarrone, A. L.; Santos, M.; Pohlmann, A. R. \& Guterres, S. S. - Int. J. Pharm., 341, p.215 (2007). PMid:17482392. http://dx.doi.org/10.1016/j. ijpharm.2007.03.031

14. Guterres, S. S.; Alves, M. P. \& Pohlmann, A. R. - Drug Target Insights, 2, p.147 (2007). PMid:21901071 PMCid:PMC3155227.

15. Sinha, V. R.; Bansal, K.; Kaushik, R.; Kumria, R. \& Trehan, A. - Int. J. Pharm., 278, p.1 (2004). PMid:15158945. http:// dx.doi.org/10.1016/j.ijpharm.2004.01.044

16. Bouchemal, K.; Briançon, S.; Perrier, E. \& Fessi, H. - Int. J. Pharm., 280, p.241 (2004). PMid:15265563. http://dx.doi. org/10.1016/j.ijpharm.2004.05.016

17. Moraes, C. M. - "Preparo, caracterização físico-química e avaliação da estabilidade de nanopartículas poliméricas contendo anestésicos locais", Dissertação de Mestrado, Universidade Estadual de Campinas, Brasil (2009).

18. Castro, M. L. \& Wang, S. H. - Polym. Bull., 51, p.151 (2003).

19. Fessi, H.; Puisieux, F.; Devissaguet, J. P.; Ammoury, N. \& Benita, S. - Int. J. Pharm., 55, p.R1 (1989). http://dx.doi. org/10.1016/0378-5173(89)90281-0

20. Külkamp, I. C.; Paese, K.; Guterres, S. S. \& Pohlmann, A. R. - Quim. Nova, 32, p.2078 (2009). http://dx.doi. org/10.1590/S0100-40422009000800018
21. Santos, E. P. - "Avaliação dos amidos hidrolisados, aniônico e catiônico, como tensoativos nas formulações de nanopartículas poliméricas para aplicação em filtros solares", Dissertação de Mestrado, Universidade de São Paulo, Brasil (2011).

22. Santos-Magalhães, N. S.; Pontes, A.; Pereira, V. M. W. \& Caetano, M. N. P. - Int. J. Pharm., 208, p.71 (2000). http:// dx.doi.org/10.1016/S0378-5173(00)00546-9

23. Schaffazick, S. R.; Pohlmann, A. R.; Freitas, L. L. \& Guterres, S. S. - Acta Farm. Bonaerense, 21, p.99 (2002).

24. Roggia, I. - "Avaliação da estabilidade, liberação $e$ permeação cutânea de nanocápsulas contendo benzofenona-3", Dissertação de Mestrado, Centro Universitário Franciscano, Brasil (2009).

25. Durán, N.; Mattoso, L. H. C. \& Morais, P. C. - "Nanotecnologia - Introdução, preparação e caracterização de nanomateriais e exemplos de aplicação", Artliber, São Paulo (2006).

26. Barrios, J. G. - "Desenvolvimento e caracterização de suspensões contendo nanocápsulas de adapaleno com diferentes núcleos oleosos", Dissertação de Mestrado, Centro Universitário Franciscano, Brasil (2010).

Enviado: $25 / 06 / 11$

Reenviado: $23 / 07 / 12$ Aceito: $14 / 08 / 12$ 